\title{
Subgrouping in Chronic Fatigue Syndrome Based on Actigraphy and Illness Severity
}

\author{
Mariya Zaturenskaya ${ }^{1}$, Leonard A. Jason ${ }^{*}, 2$, Susan Torres-Harding ${ }^{3}$ and Warren W. Tryon ${ }^{4}$ \\ ${ }^{1}$ University of Cincinnati, USA, ${ }^{2}$ DePaul University, Center for Community Research, DePaul University, 990 W. \\ Fullerton Ave., Chicago, IL 60614, USA, ${ }^{3}$ Roosevelt University, USA, ${ }^{4}$ Fordham University, USA
}

\begin{abstract}
Participants with chronic fatigue syndrome were categorized into subtypes based on actigraphy and illness selfreport symptom severity data. Each method identified two groups of patients, one with severe and one with less severe manifestations of the illness. For both subtypes, those in the more severe category had more physical functioning problems than those in the less severe categories. However, for the illness self-report symptom group, those in the more severe category had significantly more impairment in sleep, anxiety, depression, and pain, and more concurrent psychiatric status and Fibromyalgia than those in the less severe category. In contrast, those in the more severe actigraphy subtype group in comparison to the less severe group had more impairment in quality of life and cortisol readings. These findings suggest that CFS subtypes based on symptom severity and amount of activity identify different groups of patients with varying types of impairments.
\end{abstract}

Keywords: Chronic fatigue syndrome, subtyping, actigraphy, illness severity.

\section{INTRODUCTION}

Chronic Fatigue Syndrome (CFS) is a complex illness that affects about 800,000 Americans [1] and involves severe, prolonged fatigue, as well as symptoms in neurological, immunological, hormonal, gastrointestinal, musculoskeletal, and endocrinological areas [2]. Individuals with CFS vary greatly in terms of types and severity of symptoms, psychiatric comorbidity, and cognitive and physical functional status, creating a very diverse population. Heterogeneity is a major issue in CFS research and is most likely responsible for many inconsistencies and contradictions in research findings, which are especially sensitive to issues of participant selection [3].

Several research groups emphasized the importance of finding distinct subgroups within the CFS population [4, 5]. The group that developed the International criteria for CFS stressed the need to reduce heterogeneity and encouraged researchers to develop stratification strategies that would help to identify distinctive subtypes [4]. Other research groups have suggested that as with other disorders such as cancer, there are distinctive types of CFS. Thus, combining all individuals who meet the criteria for CFS prevents the identification of specific biological markers of the distinct subgroups [6].

Several researchers have suggested that distinct subgroups can be created based on symptom severity, functional level, and psychiatric status. For example, in a community-based sample study, Jason et al. [3] found that individuals who experienced a high number of symptoms of

*Address correspondence to this author at the Center for Community Research, DePaul University, 990 W. Fullerton Ave., Chicago, IL 60614, USA; E-mail: LJASON@depaul.edu severity 40 or higher on a 100 point scale, were disproportionately female, older, were more disabled and more work impaired. Hickie et al. [7] found two distinctive subtypes within their sample. Class I, the larger of the two subgroups, experienced fewer symptoms and reported their symptoms as less severe. This group had a larger proportion of males, reported a shorter duration as well as less severe course of the illness and reported less disability. Class II, the smaller subgroup reported most of the symptoms as frequent and severe, and reported greater disability and a greater percentage employed.

Other research teams have suggested the importance of illness severity as a critical variable. In their review of prognostic studies, Joyce et al. [8] reported that individuals with severe markers of CFS such as chronic symptoms, higher frequency of physical symptoms, more severe fatigue and severe disability tended to have worse prognosis, although such relationships were not always consistent. Pheley and associates [9] found that although full recovery from CFS was infrequent, individuals with less severe cases and less severe fatigue at the initial clinic visit were more likely to have a better prognosis. Janal, Ciccone, and Natelson [10] employed principal components analysis of the 10 minor symptoms of CFS, and found that disability was increased in those with the highest scores on any of their three subtypes. Depression and anxiety did not increase with the severity of symptoms. Peckerman and associates [11] determined that individuals with severe CFS had significantly lower cardiac output compared to individuals with less severe CFS. Finally, Ojo-Amaize et al. [12] found that natural killer cell activity decreased with increased severity of CFS. In summary, these studies suggest that CFS severity could be an important factor in sub-classifying the sample. 
The purpose of the present study was to compare and evaluate two different ways of stratifying a sample based on illness severity. Two strategies were used to separate the sample into a more severe subgroup and a less severe subgroup. The first strategy used self-reported data of symptoms severity for stratifying the sample into two groups. The second strategy used actigraphy, an objective measure of daily physical activity, to determine more severe and less severely ill participants within the sample. We hypothesized that the more severe group will score worse on psychosocial measures and disability/physical functioning measures.

\section{METHODOLOGY}

\section{Participant Recruitment}

Participants were recruited from a variety of sources, including physician referrals. Information about the nonpharmacologic treatment trial study was disseminated to medical colleagues through mailings, phone communication, and invited grand rounds. In addition, study announcements for new participants were placed in local newspapers and recruitment offers were made at local CFS support group meetings. These efforts were continued throughout the study period until the target enrollment numbers were achieved. One hundred and fourteen individuals were recruited.

Of the 114 individuals, $46 \%$ were referred by physicians, $34 \%$ were recruited by media (newspapers, TV, radio, etc.), and $20 \%$ stemmed from other sources (e.g., heard about the study from a friend, family member, person in the study, etc.). There were no significant demographic differences for patients recruited from these varying sources. Twenty-four additional individuals who were screened were excluded due to a variety of reasons (i.e., lifelong fatigue, less than 4 Fukuda symptoms, BMI > 45, melancholic depression or bipolar depression, alcohol or substance abuse disorder, autoimmune thyroiditis, cancer, lupus, rheumatoid arthritis). Approaches to reduce attrition included use of letters and telephone reminders of all appointments, flexibility regarding working around vacations and medical and other crises, reimbursement for transportation costs, and participant honoraria.

\section{Initial Screening}

All participants were required to be at least 18 years of age, not pregnant, able to read and speak English, and considered to be physically capable of attending the scheduled sessions. Bedridden and wheelchair bound patients were excluded due to the practical difficulties of making appointments. Referrals to local physicians who treat CFS and to support groups were offered to these individuals. After a consent form was filled out, prospective participants were initially screened by the third author, using a structured questionnaire.

\section{The DePaul CFS Questionnaire}

This screening scale was initially validated by Jason, Ropacki, et al. [13]. This scale is used to collects demographic, health status, medication usage, and symptom data, and it uses the definitional symptoms of CFS (Fukuda et al., 1994). Hawk, Jason, and Torres-Harding [14] recently revised this DePaul CFS Questionnaire, and administered the questionnaire to three groups (those with CFS, Major Depressive Disorder, and healthy controls). The revised instrument, which was used in the present study, evidences good test-retest reliability and has good sensitivity and specificity.

The DePaul CFS Questionnaire was designed to assess the diagnostic criteria for CFS as specified by Fukuda et al. [4]. For each symptom, participants were asked to indicate if the symptom had been present for 6 months or longer, if the symptom began before the onset of their fatigue or health problems, and how often (never, seldom, often/usually, or always) the symptom is experienced. Participants were also asked to rate the intensity of each symptom they endorsed on a scale of 0 to 100 , where $0=$ no problem and $100=$ the worst problem possible. This is a numerical rating scale (NRS), which has been shown to be a consistently valid measure of symptom intensity, particularly for pain intensity [15]. To measure the Fukuda et al. [4] case definition symptoms, items were designed to measure the presence of the eight minor symptoms (i.e., impaired memory or concentration, sore throat, tender lymph nodes, muscle pain, multi-joint pain, new headaches, unrefreshing sleep, and post-exertion malaise) as specified by the Fukuda et al. case definition.

\section{Psychiatric Interview}

The Structured Clinical Interview for DSM-IV (SCID) [16] Axis I was used to establish psychiatric diagnoses. The professionally administered SCID allows for clinical judgment in the assignment of symptoms to psychiatric or medical categories, a crucial distinction in the assessment of symptoms that overlap between CFS and psychiatric disorders, e.g., fatigue, concentration difficulty, and sleep disturbance [2]. A psychodiagnostic study [17] validated the use of the SCID in a sample of CFS patients. Because CFS is a diagnosis of exclusion, prospective participants were screened for identifiable psychiatric and medical conditions that may explain CFS-like symptoms. These measures were completed at DePaul University and took approximately two hours. After the initial interview was completed, the patients' information was reviewed to ensure that they met all eligibility requirements. If an individual was eligible for the study, a medical appointment was set up. Conversely, if an individual was not eligible, we discussed with him or her alternate treatment options.

\section{Medical Assessment of CFS}

The physician screening evaluation included an in-depth medical and neurological history, as well as general and neurological physical examinations. The evaluation also included a structured instrument, a modified version of the CFS questionnaire [18]. This instrument assesses the signs, symptoms, and medical history to rule out other disorders. Relevant medical information was gathered to exclude possible other medical causes of chronic fatigue, including exposure histories to tuberculosis, AIDS, and non-AIDS sexually transmitted diseases. Information on prescribed and illicit drug use was also assessed and recorded. With adult females, results of recent Pap smears and mammograms were obtained. Finally the histories of all symptoms related to CFS were gathered. 
Laboratory tests in the battery were the minimum necessary to rule out other illnesses [4]. Laboratory tests included a chemistry screen (which assesses liver, renal, and thyroid functioning), complete blood count with differential and platelet count, erythrocyte sedimentation rate, arthritic profile (which includes rheumatoid factor and antinuclear antibody), hepatitis B, Lyme Disease screen, HIV screen and urinalysis. A tuberculin skin test was also performed. Finally, an anterior-posterior and lateral chest x-ray was conducted if one had not been obtained within eight months of the study. The project physician performed a detailed medical examination to detect evidence of diffuse adenopathy, hepatosplenomegaly, synovitis, neuropathy, myopathy, cardiac or pulmonary dysfunction.

\section{Salivary Cortisol}

Individuals also completed 5 samples of salivary cortisol. Saliva was collected using Salivettes ${ }^{\circledR}$ brand collection tubes. Over the course of one day, samples were collected immediately upon first awakening and 45 minutes afterward; and at 9 AM, 4 PM, and 9 PM. The kit consists of cotton swabs inside small plastic tubes, which are placed into a storage container. Patients were instructed how to properly collect saliva samples. They first were shown how to place the cotton swab in their mouth and gently chew for 30-45 seconds. Participants were then instructed to deposit the moistened swab into its plastic tube and the tube into the container. The container recorded the exact time that they placed the plastic tube into it. Samples were held at $-20^{\circ} \mathrm{C}$ prior to assay and shipped on dry ice to Dr. Kevin Maher at the University of Miami for laboratory analysis. On the day of assay, samples were thawed, vortexed and centrifuged at 1500 RPM for 15 minutes. Salivary cortisol was determined by immunoassay using the Salimetrics high sensitivity kit (State College, PA). This kit is designed to measure cortisol levels in saliva with the calibrator in a saliva-like matrix. A built-in $\mathrm{pH}$ indicator warns the technologist of acidic or basic samples. Cortisol values from samples with $\mathrm{pH}$ values $\leq 3.5$ or $>9.0$ may be artificially inflated or lowered. This assay was run using the Biomek 2000 robotic system. Control samples with high and low concentrations of cortisol were included in each assay. Using this assay, the AM range for healthy adults is 0.94 to $1.551 \mu \mathrm{g} / \mathrm{dL}$. The PM range is from not detectible (i.e., below sensitivity of the assay) to $0.359 \mu \mathrm{g} / \mathrm{dL}$. Each participant's individual daily cortisol results were examined by an independent physician who was blinded to the identity of the study participant. The physician, Dr. Tony Lu, who is board-certified in internal medicine, then classified whether individuals had abnormal or normal cortisol levels using clinical judgment (e.g., scores over the 5 testing times were considered abnormal if they continued to rise over time, they were flat over time, they were at abnormally low levels over time).

\section{Medical Outcomes Study-Short Form-36 (MOS-SF-36)}

The MOS-SF-36, a 36 item broadly-based self-report measure of functional status related to health, identifies eight health concepts as perceived by the individual [19]. Test construction studies for the SF-36, researchers [20] have shown adequate internal consistency, significant discriminate validity among subscales, and substantial differences between patient and non-patient populations in the pattern of scores. The SF-36 has also indicated sufficient psychometric properties as a measure of functional status in a CFS population [21]. The MOS Physical Composite Index was utilized in the present investigation as combined measure of global impairment of physical functioning. A higher score indicates better health or less impact of health on functioning.

\section{Fatigue Scale (FS)}

Krupp et al.'s (1989) Fatigue Severity Scale was used to measure fatigue. This scale includes 9 items rated on 7-point scales and is sensitive to different aspects and gradations of fatigue severity. Most items in the Krupp fatigue scale are related to behavioral consequences of fatigue. Previous findings have demonstrated the utility of the Fatigue Severity Scale [22] to discriminate between individuals with CFS, MS, and primary depression [23]. In addition, the Fatigue Severity Scale [22] was normed on a sample of individuals with MS, SLE, and healthy controls.

\section{Beck Depression Inventory (BDI-II)}

Because depression is the most commonly diagnosed psychiatric disorder in CFS [24], a quantitative measure of depression severity was used. Depressive symptomatology was measured with the BDI-II [25], a 21-item self-report instrument with well-established psychometric properties. This version of the BDI is more consonant with DSM-IV criteria for major depressive disorder. The BDI-II is the only depression rating scale to be empirically tested and interpreted for both depressed and non-depressed patients with CFS [26].

\section{Beck Anxiety Inventory (BAI)}

Anxiety symptoms was measured with the BAI, a 21item self-report measure with established and replicated construct validity $[27,28]$. Factor analysis of the BAI and BDI yielded a first-order factor labeled anxiety that had salient loadings for all 21 items on the BAI, but only one item on the BDI.

\section{Perceived Stress Scale}

The Perceived Stress Scale (PSS) is a four-item revised version of a previous 14-item measure of global perceived stress. The time period that this instrument measured was the previous month [29]. The authors report a coefficient alpha reliability of .72 for the four-item short version. The Total Stress score was used in the present study. It has a range from $0-16$, with higher scores measuring more stress.

\section{Brief Pain Inventory}

The Brief Pain Inventory [30] was administered to measure the intensity of pain (pain severity) and the interference of pain in the patient's life (pain interference). Higher scores indicate more severe levels of persistent pain and higher levels of interference with functioning. An example of an item from this scale follows: During the past 24 hours pain has interfered with your general activity $(0=$ does not interfere to $10=$ completely interferes). This measure exhibits adequate levels of reliability to assess pain in noncancer samples, with coefficient alphas of .70 and above. 


\section{Quality of Life Scale}

The Quality of Life Index [31] measures perceived overall quality of life among study participants. It has been used effectively with samples of individuals with CFS [32]. The Quality of Life Index measures quality of life in four major domains: health and functioning, social and economic, psychological/spiritual, and family. This instrument differs from most other measures in its acknowledgement that individuals place different priority on different aspects of life quality. Lower scores mean more overall life satisfaction.

\section{Sleep Difficulties}

Sleep disturbances were examined by using the Pittsburgh Sleep Quality Index, which was developed to measure sleep quality in psychiatric research [33]. This index measures sleep disruptions and sleep quality. There are nineteen questions (on 0-3 scale) which generate an overall score. Acceptable measures of internal homogeneity, consistency (test-retest reliability), and validity have been reported for this measure. A global PSQI score greater than 5 yielded a diagnostic sensitivity of $89.6 \%$ and specificity of $86.5 \%$ (kappa $=0.75, \mathrm{p}<0.001)$ in distinguishing good and poor sleepers [33].

\section{Actigraph}

Participants wore an actigraph for a one week period (ActiGraph System, AM7164). An actigraph is a small, light-weight, cost-efficient activity monitor that can be worn on the waist. It has a long battery life and can continuously collect data every minute of the day and night for 22 days before its memory is filled to capacity [34]. Unlike most activity monitoring devices, the actigraph is capable of recording movement intensity. The actigraph transduces activity using an accelerometer. An 8-bit analog-to-digital converter quantifies these measurements into 128 levels of positive acceleration and 128 levels of negative acceleration 10 times each second. Integration over the resulting sampling time of $0.1 \mathrm{~s}$ in combination with other details provided by Tryon and Williams [34] would result in measurement units of 1.664 milli-g/activity activity count. For simplicity, analog-to-digital (A/D) counts are retained as activity units. The average of 600 absolute A/D values was stored in memory at the end of every minute. Participants wore the actigraph on their waist at all times except for when bathing or sleeping.

\section{Severity Criteria}

Two different strategies were used to stratify our sample of participants into more and less severely ill groups. The first strategy used questions on the DePaul Fatigue Questionnaire that involved the major symptoms (sore throat, sore lymph nodes, muscle pain, joint pain, cognitive difficulties, unrefreshing sleep, post-exertional malaise, and headaches). Individuals were placed in the high severe group if they had at least 6 out of 8 definitional symptoms at a severity level of 60 or higher on a 1-100 point scale, with 100 indicating the highest level of severity. A score of 60 or above was believed to represent a severity rating of "very severe" for each symptom, and 6 out of 8 symptoms were chosen so that an individual endorsed a clear majority of the definitional symptoms. In this sample, 29 out of 114 individuals $(25.4 \%)$ met the criteria for the "more severe" case.
For the second strategy, actigraphy data were utilized. To define a more severe group, participants were divided into three categories: low-activity, moderate-activity, and highactivity. Visual examination of the groups yielded natural cut-off scores for these three groups. We expected more severely ill participants to be less active, therefore we defined the low-activity group as a more severely ill one, while moderate- and high-activity groups were defined as less severely ill participants. In this sample, 22 individuals were classified as more severely ill and 80 as less ill (actigraphy data were only available on 102 participants).

\section{RESULTS}

We first evaluated whether there were any sociodemographic differences between the two subtypes as measured by symptom self-report severity criteria and actigraph data.

Using both severity groupings, no significant differences were found between the participants in more severe and less severe categories based on age, gender, ethnicity, education level, and socioeconomic status (more information about the sample characteristics is reported elsewhere, [35]). There were 8 participants who were categorized as more severe and 63 were categorized as less severe by both methods; however, there were inconsistencies on categorization for 31 participants (17 categorized as less severe on actigraphy were categorized as more severe on self-report measures; 14 categorized as more severe on actigraphy were categorized as less severe on self-report measures), suggesting that these two criteria capture somewhat different subgroups. However, the chi-square was not significant $\left[\chi^{2}(1, N=102)=2.13, p=\right.$ 14].

\section{Symptom Severity Groupings}

As seen on Table 1, participants with more severe selfreport symptoms scored significantly worse on the Pittsburg Sleep Inventory $[t(97)=2.49, p=.02]$, Beck Anxiety Inventory $[t(102)=3.79, p<.01]$, Beck Depression Inventory $[t(98)=3.01, p<.01]$, Brief Pain Inventory $[t(106)=2.92, p<.01]$, and on the Standardized Physical Component scale of MOS-SF $36[t(108)=2.62, p=.03]$. There were no significant differences on Fatigue Severity $[t(108)=-1.14, p=.26]$, Perceived Stress $[t(110)=.25, p=$ $.80]$, Quality of life $[t(103)=.68, p=.50]$, or on the Actigraphy scores $[t(100)=.45, p=.65]$.

Participants in the more severe category in comparison to the less severe group were significantly more likely to also have an Axis I diagnosis [59\% versus 32\%; $\chi^{2}(1, N=114)=$ $6.58, p=.01]$, and significantly more likely to be diagnosed with Fibromyalgia [45\% versus $21 \% ; \chi^{2}(1, N=112)=6.50$, $p=.01]$. There were no significant differences between the more and less severe groups on abnormal cortisol readings $\left[36 \%\right.$ versus $\left.37 \% ; \chi^{2}(1, N=98)=.01, p=.93\right]$.

\section{Activity Severity Groupings}

As seen in Table 2, participants under more severe category as defined by actigraphy criteria were found to score significantly worse on: Quality of Life Scale $[t(93)=$ 2.02, $p=.05]$, Standardized Physical Component of MOSSF $36[t(98)=2.61, p=.01]$, and Actigraphy $[t(98)=2.61$, 
Table 1. Comparisons of Means between Groups Categorized Through Self-Report Criteria

\begin{tabular}{|c|c|c|c|c|c|}
\hline \multirow{2}{*}{ Self Report Scales } & \multicolumn{2}{|c|}{ More Severe } & \multicolumn{2}{|c|}{ Less Severe } & \multirow{2}{*}{ Sig. } \\
\hline & $\mathbf{M}$ & SD & $\mathbf{M}$ & SD & \\
\hline Fatigue Severity Scale & 6.22 & .63 & 6.02 & .84 & \\
\hline Perceived Stress Scale & 8.86 & 1.46 & 8.95 & 1.82 & \\
\hline Pittsburg Sleep Inventory & 9.17 & 2.53 & 7.74 & 2.40 & $*$ \\
\hline Beck Anxiety Inventory & 17.82 & 10.34 & 11.24 & 6.75 & $* *$ \\
\hline Beck Depression Inventory & 23.70 & 9.95 & 17.12 & 9.62 & $* *$ \\
\hline Brief Pain Inventory & 5.13 & 1.98 & 3.75 & 2.21 & $* *$ \\
\hline Standardized Physical Component & 24.84 & 8.61 & 28.72 & 7.56 & $*$ \\
\hline Quality of Life Scale & 64.38 & 15.82 & 66.80 & 15.62 & \\
\hline Actigraphy & 151.71 & 62.86 & 157.73 & 56.10 & \\
\hline
\end{tabular}

$* p$ value less than .05 .

$* * p$ value less than 01

$p=.01]$. There were no significant differences on Fatigue Severity $[t(96)=1.90, p=.06]$, Perceived Stress $[t(98)=.08$, $p=.94]$, Pittsburg Sleep Inventory $[t(89)=.19, p=.85]$, Beck Anxiety Inventory $[t(91)=.00, p=1.00]$, Beck Depression Inventory $[t(90)=.52, p=.61]$, or Brief Pain Inventory $[t(95)=.02, p=.99]$.

The more severe group had significantly more abnormal cortisol readings than the less severe group [ $(55 \%$ versus $\left.26 \% ; \chi^{2}(1, N=89)=5.90, p=.02\right]$. Participants in the more severe category in comparison to the less severe category were not significantly more likely to concurrently have an Axis I diagnosis [36\% versus $38 \% ; \chi^{2}(1, N=102)=.01, p=$ .92], and were not significantly more likely to be diagnosed with Fibromyalgia [27\% versus $31 \% ; \chi^{2}(1, \mathrm{~N}=100)=.10, p$ $=.75]$.

\section{DISCUSSION}

The study's major finding was that the two ways of classifying patients, one using symptom severity self-reports and the other amount of activity, identified distinct patient groups. Only on a measure of physical functioning were those in the more severe categories for both classification systems more impaired than those in the less severe categories. There were only 8 persons with more severe classifications on both systems, which was too few for meaningful statistical analysis. For the self-report symptom group, those in the more severe category compared to those in the less severe category had significantly more impairment on sleep, anxiety, depression, pain, concurrent psychiatric status and Fibromyalgia indices. In contrast, those in the more severe actigraphy groups in comparison to the less severe group had more impairment on quality of life and cortisol readings. These findings suggest that subtypes based on symptom severity and amount of activity identify groups with impairments in different areas.

Subtyping based on symptom severity measures seemed to identify patients that were more likely to have comorbid psychiatric disorders and Fibromyalgia, as well as depression, pain, anxiety, and sleep. It is understandably that the

Table 2. Comparisons of Means between Groups Categorized Through Actigraphy Criteria

\begin{tabular}{|c|c|c|c|c|c|}
\hline \multirow{2}{*}{ Self Report Scales } & \multicolumn{2}{|c|}{ More Severe } & \multicolumn{2}{|c|}{ Less Severe } & \multirow{2}{*}{ Sig. } \\
\hline & $\mathbf{M}$ & SD & M & SD & \\
\hline Fatigue Severity Scale & 6.37 & .48 & 6.01 & .85 & \\
\hline Perceived Stress Scale & 8.86 & 1.28 & 8.83 & 1.69 & \\
\hline Pittsburg Sleep Inventory & 8.00 & 2.38 & 8.12 & 2.54 & \\
\hline Beck Anxiety Inventory & 12.95 & 8.24 & 12.96 & 8.56 & \\
\hline Beck Depression Inventory & 20.40 & 9.29 & 19.06 & 10.47 & \\
\hline Brief Pain Inventory & 4.11 & 2.13 & 4.12 & 2.30 & \\
\hline Standardized Physical Component & 23.60 & 6.60 & 28.58 & 8.23 & $*$ \\
\hline Quality of Life Scale & 60.00 & 15.42 & 67.88 & 15.48 & $*$ \\
\hline Actigraphy & 78.02 & 18.76 & 177.76 & 44.39 & $* *$ \\
\hline
\end{tabular}


more severe group had more impairment on the Composite Physical Functioning scale. What is of interest is that symptom severity groups were not differentiated on fatigue severity, stress, actigraphy, cortisol abnormalities, or quality of life. It is possible that the higher symptom self-report group identifies patients with secondary issues such as Fibromyalgia and psychiatric disorders, and these patients tend to have more depression, anxiety, pain and sleep difficulties. Having more of these secondary conditions might have a negative effect on overall physical functioning among these patients. On the other hand, it is possible that by selecting more self-report symptom variables, it is likely to identify individuals who have more somatic complaints (pain, anxiety, depression) as well as psychiatric disorders.

Actigraphy is a more objective measure that identifies a somewhat different group of patients. Actigraphy identified a group of patients who had few cortisol abnormalities; the only other objective biological measure. The cortisol abnormalities suggest that the low activity levels are symptomatic of a biological disorder; not merely volitional choices. They were unlikely to have an Axis-I disorder which is compatible with theories that consider CFS to be a nonpsychiatric disorder. They were unlikely to have Fibromyalgia which is consistent with theorists who suggest CFS is a distinct disorder. They also reported the highest physical impairment and the lowest quality of life thus documenting the adverse impact CFS has on their life.

There are several limitations in this study. For example, the size of the severe group was relatively small. We could have used a median split to divide each group, but then we would not have been able to examine those we consider more impaired either through symptom self-report of through their activity. Still, there is a certain amount of arbitrariness in how we designated the more and less severe groups, and more research with larger samples is needed to validate these categories. In addition, patients who are bed bound and possibly even more impaired were less likely to participate in this study, and therefore we still know little about this group of patients. This article was primarily presented self-report data from questionnaires in the tables. In addition, the heterogeneity of the patient population and size of the groups limits the conclusions that can be drawn from this study. Finally, response bias could also explain some of the findings, as people who report more severe symptoms of CFS might also report more severe symptoms in other areas as well.

\section{CONCLUSION}

In summary, it is important to recognize that the illness known as CFS captures a relatively heterogeneous group of patients, and subtypes might allow investigators to begin to identify unique groups with similar attributes, as has occurred in other illnesses. It appears that illness severity is a good marker for these subtypes, but illness severity can be measured either through symptom self-report data or through more objective actigraphy. The self-report data tends to identify more psychosocial features, including Fibromyalgia, psychiatric comorbidity, anxiety, depression and pain; whereas the actigraphy subtyping system tends to capture primary differences in quality of life and cortisol abnormalities. More research is needed to better understand the reasons for these differences, but clearly, it is apparent that different ways to measure subtypes does capture different patient characteristics.

\section{ACKNOWLEDGEMENT}

The authors appreciate the funding provided by NIAID (grant number AI 49720).

\section{REFERENCES}

[1] Jason LA, Richman JA, Rademaker AW, Jordan KM, Plioplys AV, Taylor RR. A community-based study of chronic fatigue syndrome. Arch Intern Med 1999; 159: 2129-37.

[2] Friedberg F, Jason, LA. Understanding chronic fatigue syndrome: An empirical guide to assessment and treatment. Washington DC: Am Psychologic Assoc 1998.

[3] Jason LA, Taylor RR, Kennedy CL, et al. Chronic fatigue syndrome: Symptom subtypes in a community based sample. Women Health 2003; 37: 1-13.

[4] Fukuda K, Straus SE, Hickie I, Sharpe MC, Dobbins JG, Komaroff A. The chronic fatigue syndrome: a comprehensive approach to its definition and study. Arch Intern Med 1994; 121: 953-9.

[5] Jason LA, Corradi K, Torres-Harding S, Taylor RR, King C. Chronic fatigue syndrome: The need for subtypes. Neuropsych Rev 2005; 15: 29-58.

[6] Corradi KM, Jason LA, Torres-Harding SR. Exploratory subgrouping in CFS: Infectious, inflammatory, and other. In: Columbus A, Ed. Advances in psychology research. Hauppauge, NY: Nova Science Publishers 2006; pp. 115-27.

[7] Hickie I, Lloyd A, Wilson A, et al. Can the Chronic Fatigue Syndrome be defined by distinct clinical features? Psychologic Med 1995; 25: 925-35.

[8] Joyce J, Hotopf M, Wessely S. The prognosis of chronic fatigue and chronic fatigue syndrome: A systematic review. Q J Med 1997; 90: 223-33.

[9] Pheley AM, Melby D, Schenck C, Mandel J, Peterson PK. Can we predict recovery in chronic fatigue syndrome? Minn Med 1999; 82: 52-6.

[10] Janal MN, Ciccone DS, Natelson BH. Sub-typing CFS patients on the basis of 'minor' symptoms. Biol Psychol 2006; 73: 124-31.

[11] Peckerman A, LaManca JJ, Dahl KA, Chemitiganti R, Qureishi B, Natelson BH. Abnormal impedance cardiography predicts symptom severity in chronic fatigue syndrome. Am J Med Sci 2003; 326: 55-60.

[12] Ojo-Amaize EA, Conley EJ, Peter JB. Decreased natural killer cell activity is associated with severity of chronic fatigue immune dysfunction syndrome. Clin Infect Dis 1994; 18: 157-9.

[13] Jason LA, Ropacki JA, Santoro NB, et al. A screening scale for chronic fatigue syndrome: Reliability and validity. J Chronic Fatigue Syndr 1997; 3: 39-59.

[14] Hawk C, Jason LA, Torres-Harding S. Reliability of a chronic fatigue syndrome questionnaire. J Chronic Fatigue Syndr 2007; 13: 41-66.

[15] Jensen MP, Karoly P. Self-report Scales and Procedures for Assessing pain in Adults. In: Turk DC, Melzack R, Eds. Handbook of Pain Assessment. New York, NY: Guilford Press 1992; pp. 13551.

[16] Spitzer RL, Williams JBW, Gibbon M, First MB. Structured Clinical Interview for the DSM-IV-Non-Patient ed (SCID-NP, Version 2.0); Washington DC: American Psychiatric Press 1995.

[17] Taylor RR, Jason LA. Comparing the DIS with the SCID: Chronic fatigue syndrome and psychiatric comorbidity. Int Rev Health Psychol 1998; 13: 1087-104.

[18] Komaroff AL, Fagioli LR, Geiger AM, et al. An examination of the working case definition of chronic fatigue syndrome. Am J Med 1996; 100: 56-64.

[19] Ware JE, Sherbourne CD. The MOS 36-item short-form health survey. Med Care 1992; 30: 473-83.

[20] McHorney CA, Ware JE, Lu AW, Sherbourne CD. The MOS 36 item Short-Form Health Survey (SF-36): III. Tests of data quality, scaling assumptions, and reliability across diverse patient groups. Med Care 1994; 32: 40-66.

[21] Buchwald D, Pearlman T, Umali J, Schmaling K, Katon W. Functional status in patients with chronic fatigue syndrome, other 
fatiguing illnesses, and healthy individuals. Am J Med 1996; 101: 364-70.

[22] Krupp LB, LaRocca NG, Muir-Nash J, Steinberg AD. The fatigue severity scale: application to patents with multiple sclerosis and systemic lupus erythematosus. Arch Neurol 1989; 46: 1121-3.

[23] Pepper CM, Krupp LB, Friedberg F, Doscher C, Coyle PK. A comparison of neuropsychiatric characteristics in chronic fatigue syndrome, multiple sclerosis, and major depression. $\mathrm{J}$ Neuropsychol Clin Neurosci 1993; 5: 200-5.

[24] Friedberg F. Chronic fatigue syndrome: a new clinical application. Prof Psychol: Res Pr 1996; 27: 487-94.

[25] Beck AT, Steer RA, Brown GK. Manual for the Beck Depression Inventory-II. San Antonio, TX: Psychologic Corpor 1996.

[26] Johnson SK, DeLuca J, Natelson B. Depression in fatiguing illness: Comparing patients with chronic fatigue syndrome, multiple sclerosis and depression. J Affect Disord 1996; 39: 21-30.

[27] Hewitt PL, Norton GR. The beck anxiety inventory: A psychometric analysis. Psychol Assess 1993; 5: 408-12.

[28] Steer RA, Clark DA, Beck AT, Ranieri WF. Common and specific dimensions of self-reported anxiety and depression: a replication. J Abnorm Psychol 1995; 104: 542-5.
[29] Cohen S, Kamark T, Mermelstein R. A global measure of perceived stress. J Health Soc Behav 1983; 37: 147-53.

[30] Cleeland CS, Ryan KM. Pain assessment: the global use of the brief pain inventory. Ann Acad Med Singap 1994; 23: 129-38.

[31] Burckhardt CS, Anderson KL. The Quality of Life Scale (QOLS): Reliability, validity, and utilization. Health Qual Life Outcomes 2003; 1 : 60.

[32] Anderson JS, Ferrans CE. The quality of life of persons with chronic fatigue syndrome. J Nerv Ment Dis 1997; 185: 359-67.

[33] Buysse DJ. The pittsburgh sleep quality index: a new instrument for psychiatric practice and research. Psychol Res 1989; 28: 193213.

[34] Tryon WW, Williams R. Fully proportional actigraphy: a new instrument. Behav Res Methods Instrum Comput 1996; 28: 392403.

[35] Jason LA, Torres-Harding S, Friedberg F et al. Non-pharmacologic interventions for CFS: a randomized trial. J Clin Psychol Med Settings 2007; 14: 275-96

(C) Zaturenskaya et al.; Licensee Bentham Open.

This is an open access article licensed under the terms of the Creative Commons Attribution Non-Commercial License (http://creativecommons.org/licenses/by$\mathrm{nc} / 3.0 /$ ), which permits unrestricted, non-commercial use, distribution and reproduction in any medium, provided the work is properly cited. 